\title{
How Does the BFKL Pomeron Couple to Quarks?
}

\author{
J. Bartels $^{a}$, J.R.Forshaw ${ }^{b}$, H.Lotter $^{a}$, L.N.Lipatov $^{c} d$ 円, M.G.Ryskin ${ }^{d}$, \\ M.Wüsthoff ${ }^{a}$
}

\begin{abstract}
${ }^{a}$ II. Institut für Theoretische Physik, Universität Hamburg.
${ }^{b}$ Rutherford Appleton Laboratory, Chilton, Didcot OX11 0QX, England.

${ }^{c}$ DESY, Inst.für Hochenergiephysik Zeuthen, Platanenallee 6, 01615 Zeuthen. ${ }^{d}$ St.Petersburg Nuclear Physics Institute, 188350, Gatchina, Russia
\end{abstract}

\begin{abstract}
We investigate the coupling of the BFKL Pomeron to quarks and to colorless states. Special emphasis is given to the effective quark-quark scattering amplitude of Mueller and Tang.
\end{abstract}

1. The BFKL Pomeron [1] is now intensively used for the QCD description of small-x physics investigated at HERA and other laboratories. Whereas in many applications (e.g. the deep inelastic structure function at small $\mathrm{x}$ ) the solution to the BFKL equation is needed only for vanishing momentum transfer $t$, in some particular final states also the nonforward direction $t \neq$ 0 enters. For this more general case the solution has been found in [2], and in its derivation the conformal symmetry of the BFKL equation plays the key role. This conformal BFKL solution contains, in momentum space, $\delta$-function like terms which have no obvious connection with perturbation theory: a physical interpretation therefore seems to be obscure.

More recently Mueller and Tang [3] suggested to define a quark-quark scattering amplitude at large momentum transfer by subtracting, from the Lipatov solution, just these $\delta$-function pieces: it was argued that only after

\footnotetext{
${ }^{1}$ Alexander von Humboldt Preisträger
} 
such a subtraction the nonforward BFKL Pomeron looks like a result of QCD perturbation theory and thus allows an interpretation in this language.

So the situation of the general (i.e. nonforward) BFKL solution looks somewhat unsatisfactory: from the point of view of the conformal symmetry, it seems doubtful whether the Mueller-Tang prescription (which is not conformally invariant) represents a solution to the BFKL equation. From a more intuitive point of view, on the other hand, one would like to have a physical interpretation of the conformal solution [2], e.g. to understand how the impulse approximation emerges. An obvious way to "bridge the gap " between these two approaches is a study of physical processes, i.e. the scattering of colorless physical states. In what cases does the quark-quark scattering amplitude with the modified (à la Mueller and Tang) Pomeron lead to the same answer as the conformal solution of [2]? And if so, how are the $\delta$-function pieces of the conformal solution connected with the impulse approximation?

In this note we shall try to answer some of these questions by studying two examples of scattering of colorless states at large momentum transfer. We begin by adopting the conformal point of view and briefly reviewing the conformal solution [2] and the Mueller-Tang [3] prescription. We then discuss, using a more intuitive language, two examples of the scattering of colorless states at large momentum transfer, and we develop some understanding of when the quark-quark scattering amplitude of Mueller and Tang will give the correct answer. Returning to more formal arguments, we then demonstrate in more detail how the impulse approximation arises from the conformal solution. In the final part we say a few words on how the absence of infrared divergencies in the Mueller-Tang amplitude can be understood.

2. The solution of the homogeneous Bethe-Salpeter equation for the pomeron wave functon for arbitrary momentum transfer $t=-Q^{2}$ has the form [2]:

$$
\begin{gathered}
\Psi_{n, \nu}\left(k_{t}, Q-k_{t}\right)= \\
\int d^{2} \rho_{1} d^{2} \rho_{2} \exp i\left(k_{t} \rho_{10}+\left(Q-k_{t}\right) \rho_{20}\right)\left(\frac{\rho_{12}}{\rho_{10} \rho_{20}}\right)^{\frac{1}{2}+i \nu+\frac{n}{2}}\left(\frac{\rho_{12}^{*}}{\rho_{10}^{*} \rho_{20}^{*}}\right)^{\frac{1}{2}+i \nu-\frac{n}{2}}
\end{gathered}
$$

where $\rho_{i j}=\rho_{i}-\rho_{j}, \rho_{1}, \rho_{2}, \rho_{0}$ are the complex coordinates of the two gluons and of the pomeron in the two-dimensional transverse subspace, resp. $\gamma=$ $\frac{1}{2}+i \nu$ is the anomalous dimension of the composite operator representing the 
pomeron, and $n$ denotes its conformal spin. Both $\gamma$ and $n$ are the quantum numbers of the corresponding irreducible representation of the conformal group which is the invariance group of the BFKL equation[2]. For unitary representations $\nu$ and $n$ take real and integer values, resp.

Some time ago Mueller and Tang [3] suggested that this expression (1) might be used for calculating quark-quark scattering amplitude at non-zero momentum transfer $Q=\sqrt{-t}$. Their arguments were the following. In order to obtain the coupling of the BFKL pomeron to a quark one should simply multiply $\Psi_{n, \nu}$ in (1) with a constant (which represents the quark impact factor) and integrate the result over $k_{t}$. As it is seen from eq.(1), after the integration we obtain a $\delta^{(2)}\left(\rho_{12}\right)$-function, and therefore, formally, the pomeron does not couple to a quark. But Mueller and Tang argued, that in [2] the conformally-invariant solution (1) of the BFKL equation had been obtained under the assumption that it will be used only for the scattering of colourless particles. Therefore in the case of the quark-quark scattering generally it is not valid. Furthermore Mueller and Tang noticed that the expression (1) contains terms proportional to $\delta^{(2)}\left(k_{t}\right)$ and $\delta^{(2)}\left(Q-k_{t}\right)$, as a result of the bad behaviour of the integrand at $\rho_{1} \rightarrow \infty$ and $\rho_{2} \rightarrow \infty$, resp. These terms give vanishing contributions when the scattering of the colourless objects is considered. Therefore there seems to be some freedom of adding or subtracting such $\delta$-function terms, and Mueller and Tang suggested to use instead of eq.(1) the following expression (for simplicity we put $n=0$ ):

$$
\begin{gathered}
\Psi_{0, \nu}^{M T}\left(k_{t}, Q-k_{t}\right)=\int d^{2} \rho_{1} d^{2} \rho_{2} \exp i\left(k_{t} \rho_{10}+\left(Q-k_{t}\right) \rho_{20}\right) . \\
\cdot\left[\left|\frac{\rho_{12}}{\rho_{10} \rho_{20}}\right|^{1+2 i \nu}-\left|\frac{1}{\rho_{20}}\right|^{1+2 i \nu}-\left|\frac{1}{\rho_{10}}\right|^{1+2 i \nu}\right] .
\end{gathered}
$$

which completely removes the singular behavior in (1). Mueller and Tang argued that such a prescription is closest to perturbation theory since there are no $\delta$-function pieces coming from the usual rules. As a result of these changes, the coupling of the pomeron to a single quark line is no longer zero:

$$
\begin{gathered}
\int \frac{d^{2} k_{t}}{(2 \pi)^{2}} \Psi_{\nu}^{M T}\left(k_{t}, Q-k_{t}\right)= \\
=-\int d^{2} \rho_{1} d^{2} \rho_{2}\left(\left|\frac{1}{\rho_{10}}\right|^{1+2 i \nu}+\left|\frac{1}{\rho_{20}}\right|^{1+2 i \nu}\right) e^{i Q \rho_{20}} \delta^{(2)}\left(\rho_{12}\right)
\end{gathered}
$$




$$
=-\pi\left(\frac{Q}{2}\right)^{-1+2 i \nu} \frac{\Gamma\left(\frac{1}{2}-i \nu\right)}{\Gamma\left(\frac{1}{2}+i \nu\right)}
$$

Bearing in mind that it was the conformal symmetry of the BFKL kernel which led to the solution (1), the subtraction procedure (2) looks somewhat strange. Indeed, so far nobody has proven that both $\Psi_{\nu}$ and $\Psi_{\nu}^{M T}$ are solutions of the BFKL equation with the same eigenvalue $\omega$, and therefore the conformal invariant expression (1) is preferable. Of course, the BFKL equation may have non-conformal invariant solutions. We know only that the set of the conformally invariant solutions is complete in the space of the generalized functions, which are integrated with impact factors with some good properties. Only impact factors of colourless particles have these properties. Further, the presence of $\delta$ functions in the solution of homogeneous BFKL equation generally does not contradict perturbation theory. One should find the solution of the inhomogeneous equation by expanding it in the series over 'singular' functions (1), as it was done in[2], and verify that up to the terms which give a zero contribution for the colourless particle scattering the result is in agreement with the perturbation theory (cf. the appendix of ref. 四).

The appearence of the singular ( $\delta$-function) term in the solution (1) is related to the fact that in the leading logarithmmic $\left(\ln \frac{1}{x}\right)$ approximation the anomalous dimension $\Delta$ of the gluonic field $\phi\left(x_{i}\right)$ in the Polyakov ansatz [5] for the three point function

$$
\begin{aligned}
E_{n, \nu}\left(\rho_{10}, \rho_{20}\right) & =<\phi\left(\rho_{1}\right) \phi\left(\rho_{2}\right) O_{n, \nu}\left(\rho_{0}\right)> \\
& =\left|\rho_{12}\right|^{-2 \Delta}\left(\frac{\rho_{12}}{\rho_{10} \rho_{20}}\right)^{\frac{1}{2}+i \nu+\frac{n}{2}}\left(\frac{\rho_{12}^{*}}{\rho_{10}^{*} \rho_{20}^{*}}\right)^{\frac{1}{2}+i \nu-\frac{n}{2}}
\end{aligned}
$$

vanishes 2. For $\Delta \neq 0$ eq.(1) contains only smeared $\delta$-functions, which are not in the contradiction with the perturbation theory. In two-dimensional conformal field theories it is natural to introduce the infinitesimal dimension $\Delta$ for the field $\phi$ as a regulator: its Green's function is proportional to $\ln \left|\rho_{12}\right|$, and it can be obtained from the general conformal invariant expression $\sim$ $\left|\rho_{12}\right|^{-2 \Delta}$ by the limiting procedure $\Delta \rightarrow 0$. From this point of view the substitution $\Psi_{n, \nu} \rightarrow \Psi_{n, \nu}^{M T}$ also looks unnatural.

Returning to the quark-quark scattering amplitude, one might adopt the view that, after all, such an amplitude is not physical, and therefore one is

\footnotetext{
${ }^{2}$ In the leading logarithmic approximation the result $\Delta=0$ follows from the normalization condition and the hermiticity property of the BFKL kernel.
} 
allowed to select any prescription for this amplitude. The physical quantity is the scattering amplitude for the colourless states. One then has to reformulate the problem in terms of observable scattering amplitudes: what is the form of the hadron amplitude, in case where the momentum transfer is significantly bigger than the essential transverse momenta of the partons inside the colliding particles? One can expect that in this kinematic region the result will have the form of the impulse approximation, with the effective quark-quark scattering amplitude being averaged with the parton wave functions. Indeed, one of the results obtained in [6] (and to be reconsidered further below) confirms the Mueller-Tang recipe for the effective quark quark scattering: namely starting from the conformally invariant solution (1) (i.e without any subtraction of the type of eq.(2)), the authors found, in one of the cases they investigated, that the result has the form of the impulse approximation although, formally, only the interaction with the different quarks $\left(\rho_{1} \neq \rho_{2}\right)$ contributes to the amplitude. In the large- $t$ limit, the scattering amplitude is dominated by the $\delta$ function pieces inside (1), and the leading term has the same form as one would have obtained under the assumption that the BFKL pomeron interacts with only one quark line. On other words, one obtains the same result as given by the Mueller-Tang prescription, i.e. without taking into account the interaction with different quark lines. However, one might also expect situations where the large- $t$ behavior of the scattering amplitude of colorless objects also feels the nonsingular part of (1): in this case the simultaneous interaction of the Pomeron with different partons becomes important, and the Mueller-Tang prescription should not be applied (or has to be modified).

3. Let us now turn to the examples which are taken from [6]. It is convenient to use the notations $R=\left(\rho_{1}+\rho_{2}\right) / 2$ and $\rho=\rho_{1}-\rho_{2}$. Let $\Phi(\rho)$ be the wave function of the initial hadron, where we omit all the arguments (coordinates) except of $\rho$. As an example, one may think of the scattering of an onium state where $\rho$ is the separation between the quark and antiquark in the impact parameter plane.

We have to start from the conformal expression (1), as this is the only known solution to the BFKL equation. So we write, as the first example, the hadron-pomeron vertex $V(Q)$ as the convolution of the eigenfunction 
$\Psi_{n, \nu}=\left(\frac{\rho_{12}}{\rho_{10} \rho_{20}}\right)^{\frac{1}{2}+i \nu+\frac{n}{2}}$ with the square of the hadron wave function:

$$
V(Q)=\int d^{2} \rho d^{2} R\left(\frac{|\rho|}{\left|R+\frac{\rho}{2}\right|\left|R-\frac{\rho}{2}\right|}\right)^{1+2 i \nu} e^{i Q R}|\Phi(\rho)|^{2}
$$

where we have put $n=0$.

As it was stressed before, from the formal point of view the expression (5) has to be associated with the graph Fig.1a (i.e. the interaction with two different quarks): the coupling of $\Psi_{n, \nu}$ to a single parton line gives zero since $\Psi_{n, \nu}=0$ at $\rho=0$. Nevertheless, in the large $Q$ limit there is a contribution of eq.(5)) which comes from the points $R \rightarrow \pm \rho / 2$ where the variation of the eigenfunction is largest and has the same form as the interaction with a single parton line (graph Fig.1b). Indeed, if $\rho>>\Delta R \sim 1 / Q$ the integration near the point $R \rightarrow \rho / 2$ gives

$$
\begin{aligned}
\int d^{2} R\left(\frac{|\rho|}{\left|R+\frac{\rho}{2}\right|\left|R-\frac{\rho}{2}\right|}\right)^{1+2 i \nu} e^{i Q R} & \simeq \int d^{2} \rho^{\prime}\left(\frac{1}{\rho^{\prime}}\right)^{1+2 i \nu} e^{i Q \rho / 2+i Q \rho^{\prime}} \\
& =\frac{\pi}{(Q / 2)^{1-2 i \nu}} e^{i Q \rho / 2} \frac{\Gamma(1 / 2-i \nu)}{\Gamma(1 / 2+i \nu)}
\end{aligned}
$$

The same result would have been obtained if we had used the Mueller-Tang prescription and taken into account only the diagram Fig.1b (note that the Mueller-Tang subtraction has a negative sign. It reflects the opposite sign of the colour coefficients corresponding to the diagrams Fig.1a and Fig.1b).

The interpretation of this contribution as representing the interaction of the BFKL Pomeron with a single quark at the point $\rho_{1}=\rho / 2$ is based upon its $Q$ dependence. We take the point of view that the $Q$-dependence is the best (maybe even the only) way to distinguish between the contributions of Fig.1a and 1b. For the impulse approximation (Fig.1b) one expects an expression of the form

$$
\int d^{2} \rho \Phi^{*}(\rho) e^{i Q \rho / 2} \Phi(\rho)=F(Q)
$$

\footnotetext{
${ }^{3}$ By impulse approximation we mean the approximation in which the interaction a compound system of several partons can be described as a sum of interactions with each parton seperately.
} 
where $F(Q)$ is the hadron form factor which vansishes for large $Q$. In contrast to this, Fig.1a is described by the following function:

$$
\left.\int d^{2} \rho \Phi^{*}(\rho)\right) e^{i(Q-k) \rho / 2-i k \rho / 2} \Phi(\rho)
$$

i.e. the appearance of a $k$-dependent exponential signals that both the quark and the antiquark share the transverse momentum $Q$. Our discussion above then implies that the conformal Pomeron (1), when coupled to (8) and restricted to the region of integration $R= \pm \rho / 2$, produces the exponential $e^{i \pm Q \rho / 2}$ and is interpreted as being associated with graph Fig.1b.

So we can proceed in two equivalent ways:

a) either we use the 'conformal' pomeron wave functions $\Psi^{n, \nu}$ (as we have done). In this case the convergence of the $\rho_{i}$ integrals is provided by the external wave function $\Phi$. As an additional advantage, this procedure also slightly reduces the number of graphs (no coupling to the single parton line). But in this way the physical interpretation in terms of Feynman diagrams becomes more difficult, and it becomes harder to exploit our physical intuition.

b) Alternatively, we could perform the Mueller-Tang subtractions in ALL the graphs Figs.1a and b (the sum of all subtraction terms gives zero due to the colourless of the initial state (hadron)). Now the integrand falls down with $\rho$, even without invoking any $\Phi$-function, and we get back the simple physical interpretation of the Feynman graphs. This method seems to be most useful in the case when one expects that the dominant contribution comes from the impulse approximation diagram: then the Mueller-Tang prescription provides a crucial simplification. The accuracy to which the impulse approximation may hold is controlled by the parameter $1 /(Q \rho)^{2}$ : if the essential $\rho>>1 / Q$ one can neglect the coupling to the different parton lines; the corrections to the impulse approximation result are of the order of $O\left(\frac{1}{Q^{2} \rho^{2}}\right)$.

So we are lead to ask the question which part of the $\rho$ integration gives the dominant contribution. In our previous example (5) the parameter $1 /(\rho Q)^{2} \sim 1$, as the typical $\rho$ in the integral is of about $1 / Q$ due to the exponent $e^{i Q \rho / 2}$. Even more, the contribution which corresponds to the coupling to different lines (Fig.1a) and comes from the "corrections" $1 /(\rho Q)^{2}$ turns out to be larger. The explanation is that the transfered momentum $Q$ can be balanced $(k \simeq Q / 2)$, and one gets a result which does not decrease 
with $Q(k=Q / 2+l)$ :

$$
\begin{aligned}
\int d^{2} \rho|\Phi(\rho)|^{2} \exp (i(Q-k) \rho / 2-i k \rho / 2) d^{2} k & \approx \int d^{2} \rho|\Phi(\rho)|^{2} \exp (-i l \rho) \\
& =\int F(2 l) d^{2} l=\mathrm{const}
\end{aligned}
$$

instead of the form factor $F(Q)$ which falls down steeply at large $Q$. So for the example (5) the impulse approximation does not give the leading contribution, and the use of the M-T prescription is not very useful.

However it is possible to change the situation in such a way that the large $\rho$ region dominates: $\rho \gg 1 / Q$. Instead of the hadron-Pomeron vertex (5) one has to consider an inclusive process in which the initial hadron is destroyed (see fig.2). In this case we get, in the expression for the cross section, two different integrals in $R$ and $R^{\prime}$ (one for the amplitude $A$, one for the complex conjugate $A^{*}$ ), but still only one integral over the parton-parton separation $\rho$. Instead of (5) we now have f:

$$
I(Q)=\int d^{2} \rho|\Phi(\rho)|^{2} \frac{|\rho|}{|R-\rho / 2||R+\rho / 2|} \frac{|\rho|}{\left|R^{\prime}-\rho / 2\right|\left|R^{\prime}+\rho / 2\right|} e^{i Q R-i Q R^{\prime}}
$$

The contributions from the "singular" points $R=R^{\prime}= \pm \rho / 2$ (or, in other words, the "Mueller-Tang terms" $\left(-1 / \rho_{1}-1 / \rho_{2}\right)$ in the Feynman diagram which describe the pomeron coupling to the single parton line) now do not have the "dangerous" exponents $\exp (i Q \rho / 2)$. Thanks to their opposite signs the exponents of $\exp (i Q R)$ and $\exp \left(-i Q R^{\prime}\right)$ cancel each other, and the result takes the form:

$$
I(Q) \simeq\left(\frac{2 \pi}{Q}\right)^{2} 2 \int d^{2} \rho|\Phi(\rho)|^{2}
$$

(the factor 2 counts the two contributions from the points $R=R^{\prime}=-\rho / 2$ and $R=R^{\prime}=\rho / 2$ ). In the graph of Fig.2 the lower part represents, at large $Q$, the structure function (say, $x G(x, Q)$ ) which in the LLA contains the logarithm $\int_{\mu^{2}}^{Q^{2}} d^{2} q / q^{2}$. In the $\rho$ representation it means that $|\Phi(\rho)|^{2} \propto 1 / \rho^{2}$ and the integral (11) over $\rho$ takes the form $\int_{1 / Q}^{1 / \mu} d^{2} \rho / \rho^{2}$ where the typical

\footnotetext{
${ }^{4}$ For symplicity we put here $\nu=0$, which is the dominant value in the high energy limit.
} 
$\rho \sim \sqrt{1 / Q \mu}>>1 / Q$. So the essential values of $\rho$ in (11) are large, and the correction $\sim 1 /(\rho Q)^{2}$ is small indeed.

Thus we presented an example where the Mueller-Tang prescription works. The criterion is the following. If the distance between the "active" (i.e. interacting with the pomeron) quark and the spectators is large in comparison with the inverse momentum transfer, the M-T prescription can be used. Such a condition can be fulfilled in inclusive processes, but not in the exclusive scattering (elastic, photoproduction or electroproduction of a vector meson). In the latter case the separation between the quarks is determined by the momentum transfer $Q$; the M-T prescription has to be generalized as stated above, and it does not simplify the calculations. The coupling to different parton lines becomes important, and method (a) involving the 'conformal' pomeron function needs fewer diagrams. In any case, one has to check whether the "distance" criterion is satisfied or not.

4. Let us take a closer look at the conformal Pomeron and see how it manages to reproduce the impulse approximation. We consider the same examples as before, i.e. the scattering of colorless states of two quarks in the large- $t$ limit. To be definite, let us start with the coupling of the pomeron to the $J / \Psi$ production vertex with a virtual photon as initial particle (figs.1a and $1 \mathrm{~b}$ ). The momentum transfer $t$ is assumed to be fixed and large compared to the virtuality of the photon $Q_{\gamma}^{2}$ and the mass of the $J / \Psi, M^{2}$. Due to the large mass of the heavy quarks the nonrelativistic approximation of the meson wave function can be applied which leads to a simple formfactor at the upper photon-meson vertex [6]. The virtual photon dissociates is a quark antiquark system which can be represented as a wave function depending on the distance $\rho=\rho_{1}-\rho_{2}$ of the two quarks. Instead of eq.(1) we use the mixed representation

$$
\Psi_{0, \nu}(\rho, Q)=\int d^{2} \rho_{2} \frac{\rho^{1+2 i \nu}}{\left[\left(\rho+\rho_{2}\right)^{2} \rho_{2}^{2}\right]^{\frac{1}{2}+i \nu}} e^{i Q \rho_{2}}
$$

The wave function of the quark-antiquark system was found to be $K_{0}\left(Q_{\|} \rho\right) e^{i Q / 2 \cdot \rho}$ $\left(Q_{\|}^{2}=\left(Q_{\gamma}^{2}+M^{2}\right) / 4\right.$, see [6]), and the analytic expression of the production vertex is:

$$
\int d^{2} \rho K_{0}\left(Q_{\|} \rho\right) e^{i Q / 2 \cdot \rho} \Psi_{0, \nu}(\rho, Q)
$$

Only fig. 1a contributes whereas $1 \mathrm{~b}$ is zero as was already discussed before. Mainly due to the phase factor $e^{i Q / 2 \cdot \rho}$ in eq (6), which is a consequence of 
the bound final state and which leads to a formfactor, a simple factorization of the wave function is impossible and the Mueller Tang prescription fails. We insert the Mellin transformed of $K_{0}$ with $\lambda$ as a real variable and derive from eq.(6) the following expression:

$$
\begin{aligned}
& 4 \pi^{4} \frac{(Q / 2)^{-3+2 i \nu_{1}}}{\Gamma^{2}\left(\frac{1}{2}+i \nu\right)} \int_{-\infty}^{\infty} \frac{d \lambda}{\pi}\left(\frac{Q^{2}}{4 Q_{\|}^{2}}\right)^{3 / 2+i \lambda} \Gamma^{2}\left(\frac{3}{2}+i \lambda\right) \\
& \frac{\Gamma\left(\frac{1}{2}-i \nu\right) \Gamma\left(\frac{1}{2}+i \nu\right) \Gamma(-i \lambda-i \nu) \Gamma(-i \lambda+i \nu)}{\Gamma\left(1+\frac{i \lambda+i \nu}{2}\right) \Gamma\left(1+\frac{i \lambda-i \nu}{2}\right) \Gamma\left(1-\frac{i \lambda+i \nu}{2}\right) \Gamma\left(1-\frac{i \lambda-i \nu}{2}\right)} .
\end{aligned}
$$

Having a closer look at eq.(7) we find a double pole in the complex $\lambda$ plane at $\lambda=3 / 2 i$. This double pole leads to a $\log \left(Q^{2} / Q_{\| 1}^{2}\right)$ in addition to the basical power behaviour $Q^{-3}=(-t)^{-3 / 2}$. A more detailed analysis shows that the momentum distribution of the two gluons is symmetric, i.e each gluon carries roughly a half of the total momentum $Q$. Moving the contour of integration beyond this pole we collect all the nonleading contributions which contain extra powers in $Q_{\|}^{2} / Q^{2}$.

The second case concerns the coupling of two pomerons (conformal dimensions $\nu_{1}$ and $\nu_{2}$ ) to one quark-antiquark system which originates from the onium state (fig.2c). The smallest scale $Q_{0}^{2}$ is given by the size of the onium and is supposed to be much smaller than $-t$. This configuration corresponds to the onium dissociation (figs.2a and $2 \mathrm{~b}$ ) into an open quark-antiquark system. The analytical expression to be calculated is (see also ref. [7]):

$$
\int d^{2} \rho\left|\Phi_{\text {onium }}(\rho)\right|^{2} \Psi_{0, \nu_{1}}(\rho, Q) \Psi_{0, \nu_{2}}(\rho,-Q) \text {. }
$$

For similar reasons as in eq.(6) only fig.2c contributes. We remark that in eq.(8) an additional phasefactor is absent in contrast to eq.(6). Inserting the Mellin transform of the onium wave function analogous to the treatment of the $K_{0}$-Bessel function in eq.(6)

$$
\int_{0}^{\infty} d \rho \rho\left|\Phi_{\text {onium }}(\rho)\right|^{2} \rho^{1+2 i \lambda},
$$

we are able to factorize off the wave function. The remaining contribution from eq.(9) is:

$$
\int_{0}^{\infty} d \rho \rho^{-2-2 i \lambda} \int_{0}^{2 \pi} d \phi \Psi_{0, \nu_{1}}(\rho, Q) \Psi_{0, \nu_{2}}(\rho,-Q)
$$


The onium wave function may contain UV-singularities, especially if one takes into account radiative corrections (a multiple pole occurs at $\lambda=i / 2$ ): the factorization has to be performed in such a way that all (collinear-) singularities including those of the vertex (eq.(8)) are absorbed into the onium structure function. This procedure is very similar to the usual 'Mass factorization' where $\lambda$ is the dimensional regulator. To be more specific let us assume that the onium is a virtual photon with the scale $Q_{0}^{2}$. The photon wave function is singular at $\rho=0$ and produces one pole. Comparing to the usual deep inelastic scattering we know that the photon structure function at lowest order perturbation theory has at least one logarithm due to the point like structure of the photon. A logarithm in $Q^{2} / Q_{0}^{2}$ requires at least a double pole in $\lambda$ at the point $\lambda=i / 2$, i.e if factorization holds, we except one more pole in expression (8).

In order to evaluate expression (8) we take the Mellin transformation of both pomeron wave functions introducing the variables $\left(\lambda_{1}\right.$ and $\left.\lambda_{2}\right)$ and find:

$$
\begin{aligned}
& \frac{\pi^{2} \sqrt{\pi}}{4 \Gamma\left(\frac{1}{2}+i \lambda\right)} \frac{2^{-2 i \lambda}\left(\frac{Q}{2}\right)^{-1+2 i \nu_{1}+2 i \nu_{2}+2 i \lambda}}{\Gamma^{2}\left(\frac{1}{2}+i \nu_{1}\right) \Gamma^{2}\left(\frac{1}{2}+i \nu_{2}\right)} \int_{-\infty}^{\infty} \frac{d \lambda_{1}}{\pi} \int_{-\infty}^{\infty} \frac{d \lambda_{2}}{\pi} \Gamma\left(-i \lambda_{1}+i \nu_{1}\right) \\
& \Gamma\left(-i \lambda_{1}-i \nu_{1}\right) \Gamma\left(-i \lambda_{2}+i \nu_{2}\right) \Gamma\left(-i \lambda_{2}-i \nu_{2}\right) \frac{\Gamma\left(\frac{1}{2}-i \lambda+i \lambda_{1}+i \lambda_{2}\right)}{\Gamma\left(\frac{1}{2}+i \lambda-i \lambda_{1}-i \lambda_{2}\right)} \\
& \Gamma\left(i \lambda-i \lambda_{1}-i \lambda_{2}\right) \frac{\Gamma\left(\frac{1}{2}+i \lambda_{1}\right) \Gamma\left(\frac{1}{2}+i \lambda_{2}\right) \Gamma\left(2 i \lambda-i \lambda_{1}-i \lambda_{2}\right)}{\Gamma\left(\frac{1}{2}+i \lambda-i \lambda_{1}\right) \Gamma\left(\frac{1}{2}+i \lambda-i \lambda_{2}\right)}
\end{aligned}
$$

A careful investigation of expression (11) shows that a double pinching in $\lambda_{1}$ and $\lambda_{2}$ occurs as soon as $\lambda$ approaches $i / 2$, i.e the integrals over $\lambda_{1}$ and $\lambda_{2}$ induces a double pole in $\lambda$ at $\lambda=i / 2$. The $\Gamma(1 / 2+i \lambda)$ in the denominator in front of expression (11) reduces the double pole to a single pole. The residue is simply the factor

$$
\pi^{2}\left(\frac{Q}{2}\right)^{-2+2 i \nu_{1}+2 i \nu_{2}} \frac{\Gamma\left(\frac{1}{2}-i \nu_{1}\right)}{\Gamma\left(\frac{1}{2}+i \nu_{1}\right)} \frac{\Gamma\left(\frac{1}{2}-i \nu_{2}\right)}{\Gamma\left(\frac{1}{2}+i \nu_{2}\right)} .
$$

which is identical to the expression resulting from eq. (3). In addition, a logarithm in $Q^{2} / Q_{0}^{2}$ occurs reflecting the pointlike structure of the photon. It has to be absorbed in the photon wave function. In the case of a nonsingular onium wave function we get an overall factor $\int d^{2} \rho\left|\Phi_{\text {onium }}\right|^{2}$. 
The result (19) agrees with what one would have obtained by using only the $\delta$-function pieces of the pomeron wave function. We therefore conclude that the large- $t$ limit is governed by these singular pieces; correspondingly, the distribution of the momenta of the two gluons in the conformal pomeron is very asymmetric: one gluon is very soft whereas the other one carries the total momentum $Q$. Why do we, nevertheless, get the same result as in in the impulse approximation? The answer is very simple: the soft gluon line of the conformal Pomeron does not distinguish to which of the quark lines it couples. The coefficient of the $\delta$ function, on the other hand, is identical to the result from the impulse approximation. The result therefore looks identical to the impulse approximation and confirms the Mueller-Tang prescription.

5. The last question we would like to discuss is the infrared logarithm which is present in the two gluon exchange quark-quark amplitude (fig.3) but disappears in the asymptotic Mueller-Tang formula 3]. Why does the small $k_{t}$ (see fig.3) region do not contribute to the high energy amplitude? The answer to this question was given in ref. [6]: beginning at small energies, one can investigate, step by step, the disappearence of the infrared logarithm. As long as $z=\frac{N_{c} \alpha_{s}}{2 \pi} \ln \frac{s}{Q^{2}}<1$ it is possible to sum up the double-log terms of the type $\left(\alpha_{s} \ln \frac{s}{Q^{2}} \ln \frac{Q^{2}}{k_{t}^{2}}\right)^{n}$. These terms comes from the reggeizized part of the BFKL kernal[1], i.e. from the Feynman graphs fig.3b,c. They are easily exponentiated $(z \leq 1$ and $k<<Q)$ :

$$
f(k ; Q, z) \propto \frac{\left(k_{t}^{2} / Q^{2}\right)^{z}}{k_{t}^{2}(Q-k)_{t}^{2}}
$$

As a result of this exponentiation, the contribution of the region $k_{t}<<Q$ dies out with energy, at least up to $z \leq 1$. At larger energies this simple formula can no longer be used since subdominant terms become important which have been neglected in (7). For $z>1$ nothing dramatical happens near $k_{t}=0$, and when iterating the integral equation for the BFKL Pomeron the behaviour of the n-th iteration $f_{n}(k ; Q, z)$ at small $k<<Q$ is determined mainly by the value of the previous iteration step $f_{n-1}(k ; Q, z)$ in the region $k \sim Q / 2$. To see this absence of infrared logarithms most clearly we return to the $\rho$-representation and subtract from Lipatov's conformal solution (1) the $\delta\left(k_{t}\right)$ term, i.e. we make use of the Mueller Tang formula (2). As a result of the subtractions, infrared divergencies are absent. In the high energy limit 
typical values of $\nu$ are small $(|\nu|<1 / 2)$. In the limit $Q>>k$ the essential $\rho_{2}$-values are of the order $\sim 1 / Q$ and, after the subtraction, the essential region of $\rho_{1}$ is of the order of $\rho_{2}$; i.e. $\rho_{1} \sim 1 / Q$ too. This means that in the region $k_{t}<<Q$ the amplitude (2) becomes practically independent of $k_{t}$. In particular it does not contain the singularity $1 / k_{t}^{2}$ which in the two gluon exchange diagram 3 a leads to the infrared logarithm $\int_{k_{0}^{2}}^{Q^{2}} d^{2} k / k^{2}=\ln Q^{2} / k_{0}^{2}$ ( $k_{0}$ is the infrared cutoff).

The situation changes crucially in the case of $\nu \rightarrow i / 2$, which corresponds to the "low" energy (DGLAP) limit. From the formal point of view the expression in the square brackets still goes to zero as $\rho_{1} \rightarrow \infty$, but only slowly. Therefore we have no $\delta$-functions $\left(\delta\left(k_{t}\right)\right)$ in eq.(2). But when the power $1 / 2+i \nu$ becomes very small the square bracket is close to -1 over a very large region of $\rho_{1}$, and when integrating over this region one gets $f(k ; Q) \propto \frac{1}{k^{2}(Q-k)^{2}}$, as it should be for the two gluon exchange amplitude. This example shows how in eq.(2) the infrared logarithm (i.e. the behaviour $\left.f(k, Q) \propto 1 / k^{2}\right)$ is restored in the DGLAP limit $(\nu \rightarrow i / 2)$.

At first sight this calculation seems to give the wrong minus sign. However, this sign corresponds to the diagram fig.1a, where the two t-channel gluons couple to different quarks. On the other hand we know that the infrared logarithm comes from the Feynman graph Fig.1b where both gluons interact with the same coloured parton. Since both quarks form a colourless state the colour coefficient in Fig.1b has the opposite sign compared to Fig.1a, and the minus sign in the square brackets of eq.(8) is just a result of this color coefficient.

We hope that this discussion gives a better understanding of the conformal BFKL pomeron eigenfunctions, helps to clarify the origin of the MuellerTang prescription and, in particular, provides a criterium for its use in describing the high energy scattering of colorless states.

Acknowledgements: L.Lipatov and M.Ryskin wish to thank DESY and the II.Institut für Theoretische Physik, Universität Hamburg, for their hospitality.

This work has been supported in part by the Alexander von Humboldt Foundation (L.N.Lipatov), the Volkswagen Stiftung (M.G.Ryskin), and the Deutsche Akademische Austauschdienst DAAD (J.R.Forshaw). 


\section{References}

[1] E.A.Kuraev and L.N.Lipatov,V.S.Fadin, Sov.Phys.JETP 44 (1976) 443, Sov.Phys.JETP 45 (1977) 199,

Y.Y.Balitskij and L.N.Lipatov, Sov.J.Nucl.Phys 28 (1978) 822.

[2] L.N.Lipatov, Sov.Phys.JETP 63 (1986) 904.

[3] A.H.Mueller and W.K.Tang, Phys.Lett. B 284 (1992) 123.

[4] L.N.Lipatov, in "Perturbative QCD" (ed. A.H.Mueller), p. 411, World Scientific, Singapore 1989 (Advanced Series on Directions in High Energy Physics).

[5] A.M.Polyakov, JETP Lett. 12 (1970) 381.

[6] J.R.Forshaw, M.G.Ryskin, DESY-94-112.

[7] J.Bartels, H.Lotter, M.Wüsthoff, DESY-94-245.

\section{Figure captions}

Fig. 1a: Graphical representation of the coupling of the conformal covariant three-point function $\Psi_{0, \nu}$ to the Photon-Meson vertex. Here the gluons represented by the wavy lines couple to different quarks of the meson wavefunction.

Fig. 1b : The same as in fig.1a but with the two gluons coupling to the same quark of the meson wavefunction.

Fig. 2a : Coupling of the conformal covariant three-point function to the quark-antiquark system originating from an onium state. Gluons are coupled to different quarks of the onium wavefunction.

Fig. 2b : Same as in fig.2a but with the two gluons coupling to the same quark of the onium wavefunction.

Fig. 2c : Graphical representation of the cross section of the dissociation of an onium state into an open quark-antiquark state (cf. eqs. (10),(15)). 
Fig. 3a : The two-gluon exchange quark-quark-amplitude.

Fig. 3b : Higher order contribution to the quark-quark amplitude which is part of the reggeized part of the BFKL-kernel.

Fig. 3c: Another contribution to the reggeized part of the BFKL-kernel. 


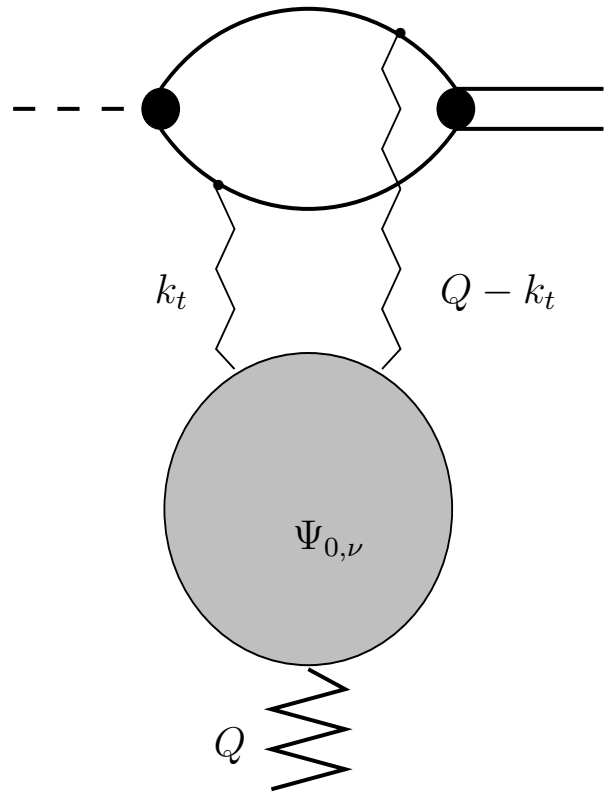

Figure 1a: 


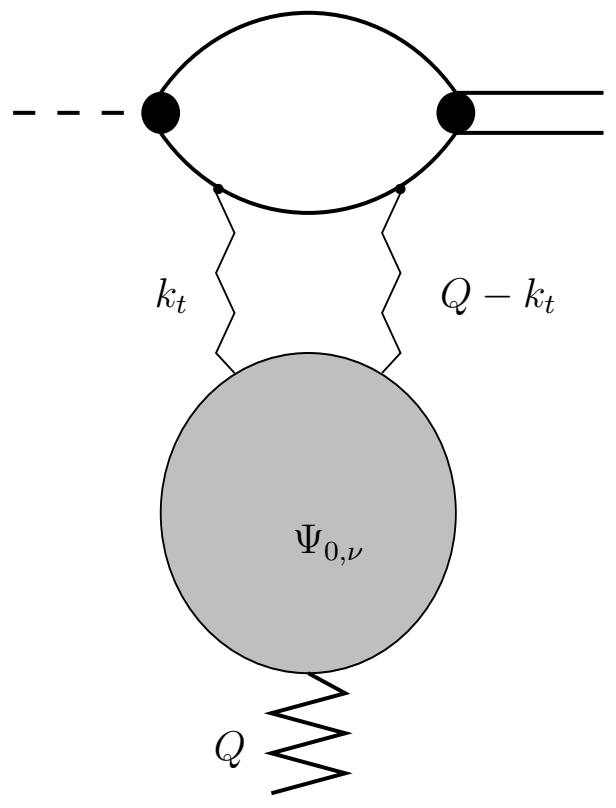

Figure 1b: 


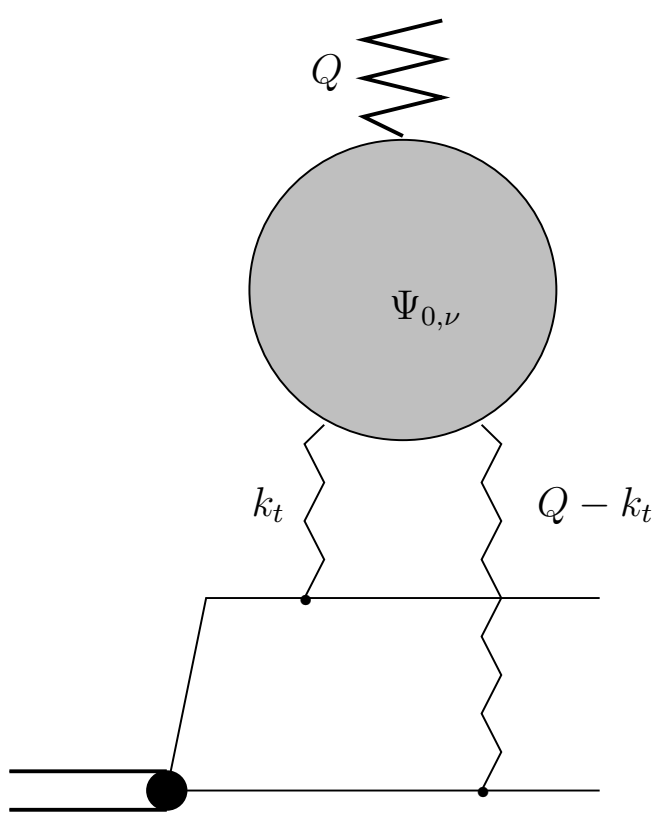

Figure 2a: 


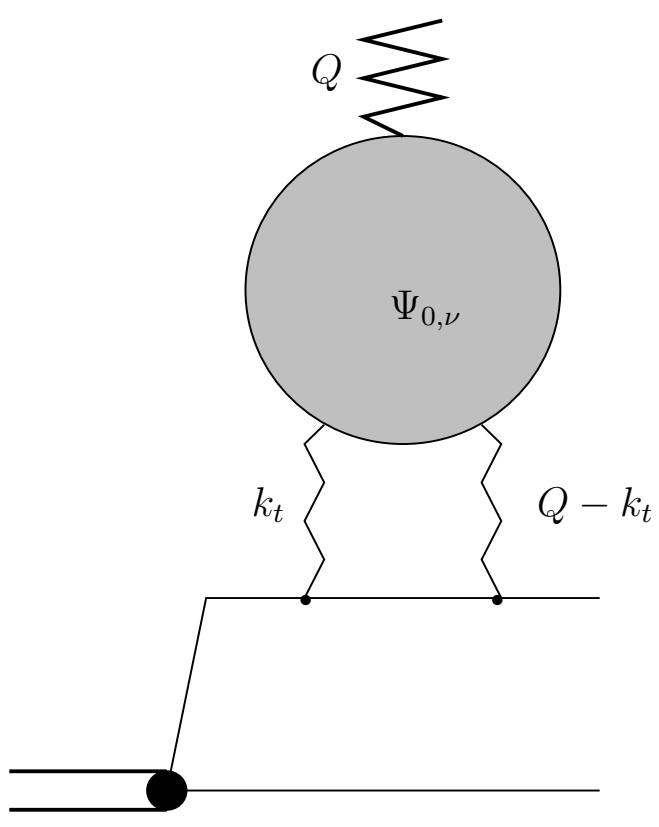

Figure 2b: 


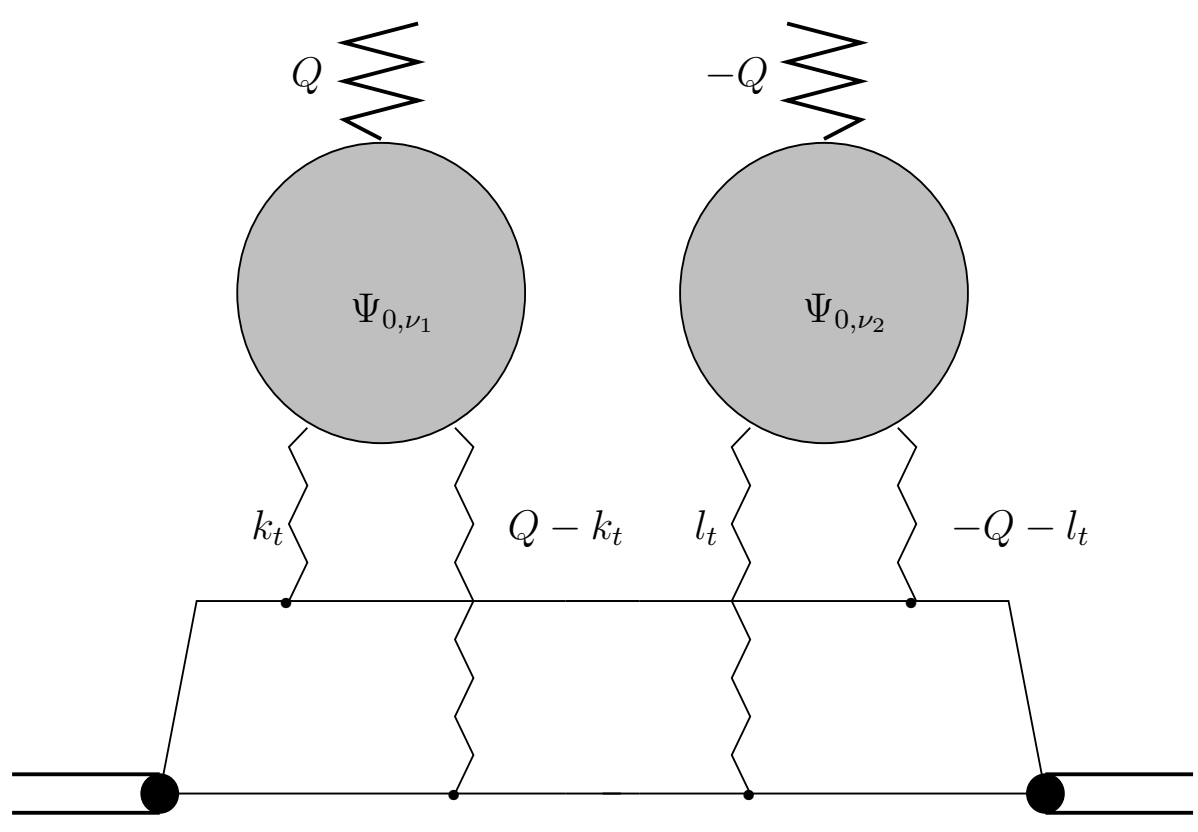

Figure 2c: 


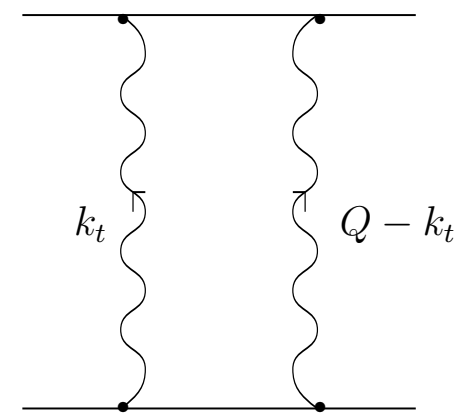

Figure 3a:

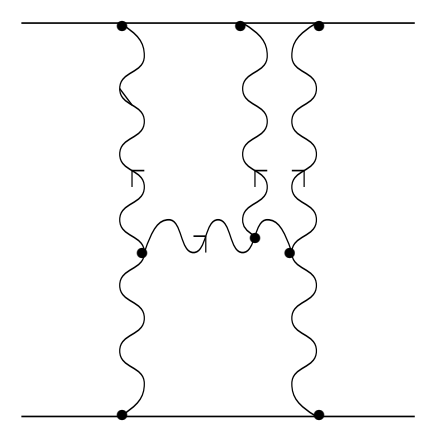

Figure 3c:

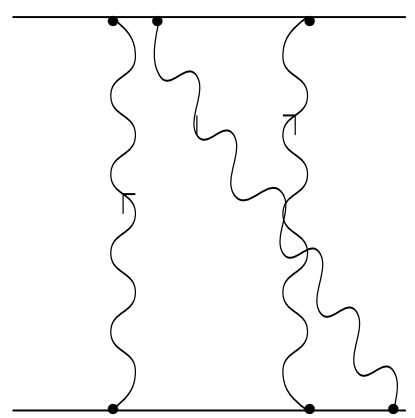

Figure 3b: 